\title{
Hepatic ferroptosis plays an important role as the trigger for initiating inflammation in nonalcoholic steatohepatitis
}

Shinya Tsurusaki ${ }^{1,2}$, Yuichi Tsuchiya ${ }^{3}$, Tomoko Koumura $^{4}$, Misaki Nakasone ${ }^{4}$, Taro Sakamoto ${ }^{4}$, Masaki Matsuoka ${ }^{4}$, Hirotaka Imai ${ }^{4}$, Cindy Yuet-Yin Kok $\mathbb{B}^{1,6}$, Hitoshi Okochi ${ }^{1}$, Hiroyasu Nakano $\mathbb{B}^{3}$, Atsushi Miyajima ${ }^{5}$ and Minoru Tanaka ${ }^{1,2}$

\begin{abstract}
Nonalcoholic steatohepatitis (NASH) is a metabolic liver disease that progresses from simple steatosis to the disease state of inflammation and fibrosis. Previous studies suggest that apoptosis and necroptosis may contribute to the pathogenesis of NASH, based on several murine models. However, the mechanisms underlying the transition of simple steatosis to steatohepatitis remain unclear, because it is difficult to identify when and where such cell deaths begin to occur in the pathophysiological process of NASH. In the present study, our aim is to investigate which type of cell death plays a role as the trigger for initiating inflammation in fatty liver. By establishing a simple method of discriminating between apoptosis and necrosis in the liver, we found that necrosis occurred prior to apoptosis at the onset of steatohepatitis in the choline-deficient, ethionine-supplemented (CDE) diet model. To further investigate what type of necrosis is involved in the initial necrotic cell death, we examined the effect of necroptosis and ferroptosis inhibition by administering inhibitors to wild-type mice in the CDE diet model. In addition, necroptosis was evaluated using mixed lineage kinase domain-like protein (MLKL) knockout mice, which is lacking in a terminal executor of necroptosis. Consequently, necroptosis inhibition failed to block the onset of necrotic cell death, while ferroptosis inhibition protected hepatocytes from necrotic death almost completely, and suppressed the subsequent infiltration of immune cells and inflammatory reaction. Furthermore, the amount of oxidized

phosphatidylethanolamine, which is involved in ferroptosis pathway, was increased in the liver sample of the CDE dietfed mice. These findings suggest that hepatic ferroptosis plays an important role as the trigger for initiating inflammation in steatohepatitis and may be a therapeutic target for preventing the onset of steatohepatitis.
\end{abstract}

\section{Introduction}

Nonalcoholic fatty liver disease (NAFLD), a kind of lifestyle disease ranging from simple steatosis to steatohepatitis, is the most prevalent liver disease in the world. Nonalcoholic steatohepatitis (NASH) is a severe form of NAFLD that is characterized by lipid droplet accumulation in hepatocytes, hepatic cell death, infiltration of

\footnotetext{
Correspondence: Minoru Tanaka (m-tanaka@ri.ncgm.go.jp)

${ }^{1}$ Department of Regenerative Medicine, Research Institute, National Center for Global Health and Medicine, Tokyo, Japan

${ }^{2}$ Laboratory of Stem Cell Regulation, Institute for Quantitative Biosciences, The University of Tokyo, Tokyo, Japan

Full list of author information is available at the end of the article

Edited by A. Oberst
}

inflammatory cells, and mostly fibrosis. While the prognosis of simple steatosis is benign, the progression into NASH is a serious risk factor for cirrhosis and carcinogenesis $^{1,2}$. Therefore, it is important to elucidate the mechanism underlying the pathological process from simple steatosis to steatohepatitis. Although the "two-hit theory" and "multiple parallel hit hypothesis" have been proposed in the pathogenesis of NASH, hepatic cell death triggered by abnormal lipid accumulation in hepatocytes is considered as a likely cause of inflammation ${ }^{3}$. Several studies have already reported that apoptosis and necroptosis are involved in the pathological process of NASH, by using cell death-related genetically modified mouse

\section{(c) The Author(s) 2019}

(c) (i) Open Access This article is licensed under a Creative Commons Attribution 4.0 International License, which permits use, sharing, adaptation, distribution and reproduction cc) in any medium or format, as long as you give appropriate credit to the original author(s) and the source, provide a link to the Creative Commons license, and indicate if changes were made. The images or other third party material in this article are included in the article's Creative Commons license, unless indicated otherwise in a credit line to the material. If material is not included in the article's Creative Commons license and your intended use is not permitted by statutory regulation or exceeds the permitted use, you will need to obtain permission directly from the copyright holder. To view a copy of this license, visit http://creativecommons.org/licenses/by/4.0/. 
models or chemical inhibitors ${ }^{4,5}$. These studies have highlighted the contribution of each type of cell death to the progression of NASH based on relatively later symptoms such as fibrosis and carcinogenesis. However, the type of cell death involved in the onset of NASH has not been investigated, as it is quite difficult to specify the timing of cell death occurring after simple steatosis in long-term disease models. As such, the initial type of cell death that acts as the trigger for inflammation, and causes the onset of steatohepatitis from steatosis remains elusive. The aim of this study was to investigate the type of hepatocyte cell death involved in the onset of steatohepatitis. To focus on the earliest stage of hepatocyte cell death just before steatohepatitis, we explored apoptosis and necrotic cell death using the choline-deficient, ethionine-supplemented (CDE) diet model, which induces steatohepatitis in a relatively short period of time ${ }^{6}$. While the methionine-deficient, choline-deficient (MCD) or choline-deficient, L-amino acid-defined (CDAA) diet is a widely used mouse model of $\mathrm{NASH}^{7-9}$, the $\mathrm{CDE}$ model utilizes the supplementation of ethionine instead of methionine deficiency, which is a methionine analog for methionine starvation. We first determined the timeframe for the onset of steatohepatitis, based on elevation of serum markers for liver damage. We then aimed to identify the initial cell death that occurred within this timeframe. We have also established a method for distinguishing apoptosis and necrosis easily in the liver and have evaluated the type of hepatocyte cell death by using cell death-related inhibitory agents and knockout mice in this model. From these experiments, we discovered the role of ferroptosis as the initial cell death that triggers steatohepatitis.

Ferroptosis is a type of "programmed necrosis" recently identified as an iron- and lipid hydroperoxide-dependent nonapoptotic cell death in cancer cells. It has been reported that iron chelators as well as a few genes related to the lipid peroxide removal system such as cystine/glutamic acid transporter $(\mathrm{xCT})$ and glutathione peroxidase 4 $(\mathrm{GPx} 4)$ are involved in the suppression of ferroptosis ${ }^{10,11}$. It is also reported that hydroperoxidation of phosphatidylethanolamine (PE) within the lipid membrane causes induction of ferroptosis ${ }^{12,13}$. Currently, there is accumulating evidence that ferroptosis is involved in not only cell death of a certain type of tumor cells but also in the pathogenesis of several diseases such as neuronal dysfunction and acute kidney injury ${ }^{14,15}$. However, the role of ferroptosis in liver disease remains poorly understood.

In this study, we focus on the type of cell death that initially occurs at the earliest stage of NASH. Our data reveal that ferroptosis is the type of necrosis that precedes the other type of cell death, thus giving cues to initiate inflammation in this model. Our findings suggest the role of ferroptosis as a trigger at the onset of steatohepatitis.

\section{Results}

\section{Pathological feature of liver injury after the CDE diet feeding}

The CDE diet is widely used as a murine chronic liver injury model that induces steatohepatitis and expansion of liver progenitor cells ${ }^{16}$. To characterize the pathological features of this model, we first investigated the status of $\mathrm{NASH}$ symptoms, i.e. fat accumulation, hepatocyte cell death, infiltration of immune cells, and fibrosis after the $\mathrm{CDE}$ diet feeding. The Oil Red $\mathrm{O}$ staining revealed that the increased fat deposition in hepatocytes was evident 1 day after CDE diet feeding and that drastic accumulation of lipid droplets was observed by 2 days after feeding (Fig. 1a). Next, we measured serum liver injury markers, aspartate aminotransferase (AST) and alanine aminotransferase (ALT) at several time points after the CDE diet feeding. Because hepatocytes are abundant in AST and ALT, the release of these two enzymes into serum represents the degree of hepatocyte cell death. As shown in Fig. 1b, the levels of both AST and ALT sharply increased after 2 days of the CDE feeding as previously reported $^{6}$. Such a sharp increase of AST and ALT values was not detected after the CDAA-based high-fat diet feeding (Supplementary Fig. S1) ${ }^{17}$. In addition, infiltration of inflammatory cells was observed in the liver by immunostaining of CD11b, and gene expression of inflammatory cytokines was also upregulated (Fig. 1c, d). Thereafter, the levels of AST and ALT were decreased and maintained at a constant level, shifting to chronic liver injury phase. Consistent with the outcome of chronic hepatitis, the liver exhibited fibrosis at day 21 as evaluated by Picro-Sirius Red staining and expression analysis of fibrosis-related genes (Fig. 1e, f). Taken together, these results suggest that an initial cell death which triggers inflammation occurs within 2 days after CDE diet feeding in this model. Therefore, we further investigated the type of cell death at an early stage of steatohepatitis.

\section{Evaluation of cell death in the liver by establishment of in vivo necrosis detection method}

While there are several methods for detecting apoptotic cells such as TUNEL, detection of single-strand DNA or activation of caspases, there is currently no method for convenient identification of necrosis in vivo. Electron microscopy is an excellent tool for distinguishing between apoptosis and necrosis; however, it is not suitable for observing several types of cell death simultaneously over a broad range of injured tissue. To establish a simple method to detect necrotic cells, we adapted the method of propidium iodide (PI) staining for in vivo study, which is widely used in vitro to identify necrosis. In necrotic cells, the collapsed plasma membrane allows PI to stain the cell nucleus, whereas it is excluded from early apoptotic cells with intact plasma membrane. To test whether this 

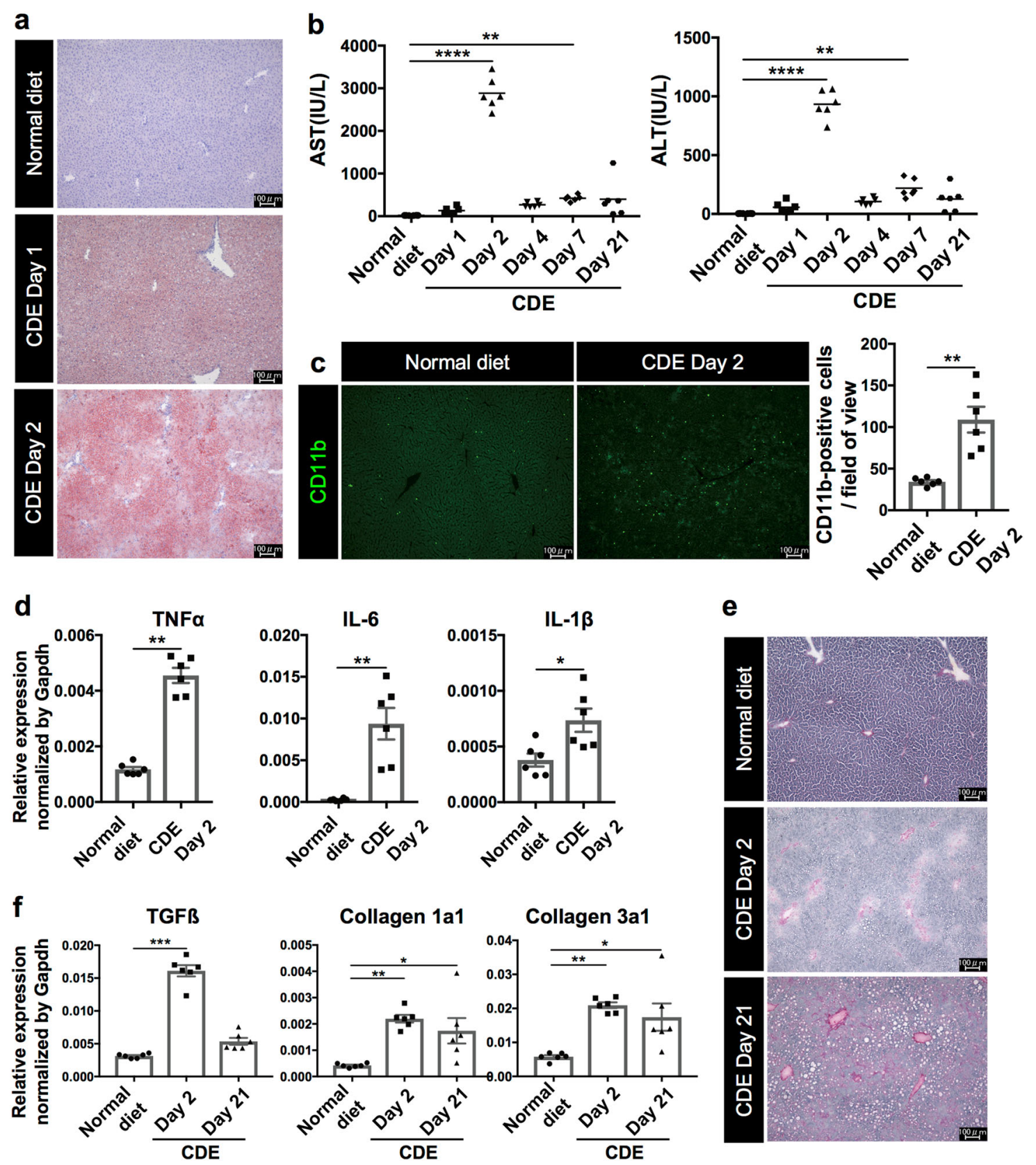

Fig. 1 Pathological features of steatohepatitis in the CDE diet model. The lipid accumulation, inflammatory responses, and fibrosis in the liver were evaluated after choline-deficient, ethionine-supplemented (CDE) diet feeding. Six mice were used for analyses at each time point. a Representative images of lipid accumulation in the liver by Oil Red O staining after 0,1 , and 2 days post CDE diet feeding. b Measurement of serum liver injury markers, aspartate aminotransferase (AST) and alanine aminotransferase (ALT) after the CDE feeding $\left(n=6\right.$; $\left.{ }^{* *} P<0.01,{ }^{* * * *} P<0.0001\right)$. c Fluorescence images of infiltration of immune cells by immunohistochemistry of liver sections for CD11b. The number of CD11b-positive cells was evaluated in 20 non-overlapping fields of view for each biological sample. The data are shown as the mean \pm SEM $(n=6$; **P<0.01). d Gene expression analysis of inflammatory cytokines by quantitative reverse transcriptase PCR (RT-PCR). All data are normalized to Gapdh and shown as the means $\pm \operatorname{SEM}\left(n=6,{ }^{*} P<0.05,{ }^{*} P<0.01\right)$. e Representative images of liver fibrosis by Picro-Sirius Red staining. $\mathbf{f}$ Expression analysis of fibrosis-related genes by quantitative RT-PCR. All data are normalized to Gapdh and shown as the means \pm SEM $\left(n=6 ;{ }^{*} P<0.05,{ }^{* *} P<0.01,{ }^{* * *} P<0.001\right)$. Scale bar $=$ $100 \mu \mathrm{m}$

principle works well in vivo, we injected PI intravenously into carbon tetrachloride $\left(\mathrm{CCl}_{4}\right)$-treated mouse, which is a typical model of necrotic liver injury ${ }^{18}$. The liver damage induced by $\mathrm{CCl}_{4}$-treatment was confirmed by elevation of serum AST and ALT levels, and hematoxylin-eosin (HE) staining showed extensive necrotic areas around the 
central veins (CV) (Fig. 2a, b). PI staining revealed that numerous PI-positive cells were observed around $\mathrm{CV}$ in this model, whereas apoptotic cells were rarely detected by immunostaining of cleaved caspase-3 (CC3) (Fig. 2c). Co-staining of PI and CC3 demonstrated that most CC3positive apoptotic cells are negative for PI (Fig. 2c). These results demonstrated that this in vivo necrosis assay can be used to detect necrotic cells. It was also possible to distinguish between apoptosis and necrosis by co-staining of PI with apoptosis-related marker in the early stages of hepatic cell death.

To identify the type of cell death at the onset of steatohepatitis, we applied the PI and CC3 staining method to the injured liver in the CDE model. Consistent with the rapid increase and subsequent decrease of serum AST and ALT (Fig. 1b) after CDE feeding, many PI-positive cells were detected in the injured liver after 2 days of $\mathrm{CDE}$ feeding, while a small number of PI-positive cells were observed 3 weeks later (Fig. 2d and Supplementary Fig. S2A). In contrast, no PI-positive cells were visible in normal liver (Fig. 2d). Immunostaining of CC3 revealed that several apoptotic cells were also detected in the same liver. However, CC3 and PI double-positive cells were hardly detected (Fig. 2d), suggesting that CC3-positive apoptotic cells and PI-positive necrotic cells existed independently in the injured liver. More intriguingly, we noticed that the initial cell death at day 2 seemed to occur predominantly at the portal region where is surrounding the portal vein and CK19-stained bile ducts (Fig. 2d). To further clarify the regional property in hepatic cell death, we compared the ratio of PI-positive cells between Cadherin1 (CDH1)positive and -negative areas in the liver, because $\mathrm{CDH} 1$ is known to be preferentially expressed in hepatocytes at the portal region ${ }^{19}$. As a result, CDH1-positive region showed significantly higher ratio than $\mathrm{CDH} 1$-negative region (Supplementary Fig. S2B), suggesting that hepatic necrosis initially begins around the portal vein after CDE feeding.

\section{Necrosis precedes apoptosis at the onset of steatohepatitis}

Since both apoptosis and necrosis had already occurred by day 2 , we next investigated the status of fatty liver at an earlier time point to determine which cell death precedes the onset of steatohepatitis. To this end, we designed a new experimental scheme as shown in Fig. 3a. To standardize the time at which feeding commenced, mice were fasted for $24 \mathrm{~h}$ prior to feeding with CDE diet. After CDE diet was commenced $(0 \mathrm{~h})$, liver samples were analyzed at $10,12,14,16$, and $18 \mathrm{~h}$ post feeding. Unlike the injured liver at day 2, apoptosis could not be detected in the injured liver by either western blotting or CC3 immunostaining from 10 to $18 \mathrm{~h}$ post $\mathrm{CDE}$ diet feeding (Fig. $3 \mathrm{~b}$, c). In contrast, necrosis was observed as early as $14 \mathrm{~h}$ post feeding and thereafter the number of necrotic cell gradually increased (Fig. 3c and Supplementary Figure S3A,
B). The PI-positive necrotic cells seemed to be mainly derived from CDH1-positive hepatocytes, although a minor contribution of CD11b-positive immune cells to necrosis was observed at $18 \mathrm{~h}$ post CDE diet feeding (Supplementary Fig. S3C, d). Consistent with this observation, the levels of serum liver injury markers and expression of tumor necrosis factor- $\alpha$ (TNF $\alpha$ ) mRNA began to increase between 12 and $14 \mathrm{~h}$ after the CDE diet feeding (Fig. 3d, e). These results indicated that necrosis occurred prior to apoptosis, and is thereafter likely to be the trigger for the onset of steatohepatitis in this model.

\section{Necroptosis is not the cell death that initiates steatohepatitis in the CDE model}

Since necrosis is likely to be a trigger of inflammation, we further investigated the type of necrosis occurring at the initiation of steatohepatitis. Firstly, we examined whether "necroptosis" was involved in the initial necrosis of this model, because necroptosis has been implicated in inflammation and fibrosis in MCD diet or high-fat diet models ${ }^{5,20}$. To address this issue, we administered a chemical inhibitor of necroptosis, Necrostatin-1s (Nec$1 \mathrm{~s})$, in four doses at $10,12,14$, and $16 \mathrm{~h}$ post CDE feeding, and then evaluated the liver at $18 \mathrm{~h}$ by measurement of serum AST, ALT, in vivo necrosis assay, and gene expression analysis of inflammatory cytokines (Fig. 4a). We chose to begin administration of Nec-1s at $10 \mathrm{~h}$ as we had observed no sign of necrosis at this time point within our experimental settings, based on normal levels of serum AST and ALT (Fig. 3d). We hypothesized that if the initial necrosis was necroptosis, the symptoms of steatohepatitis should be successfully suppressed by Nec$1 \mathrm{~s}$ administration. However, since the administration of Nec-1s failed to reduce serum AST and ALT (Fig. 4b), necroptosis is not likely to be the initial cell death in this model. Consistently, there was no apparent difference in the emergence of PI-positive necrotic cells and gene expression of inflammatory cytokines between control and Nec-1s-treated mice (Fig. 4c, d), suggesting that Nec1s could not block the initial necrosis. Next, we investigated the phosphorylation of two key regulators of necroptosis, RIPK3 and MLKL ${ }^{21}$, by western blot analysis. As a result, neither the phosphorylation of RIPK3 nor MLKL was detected in the liver samples from 10 to $18 \mathrm{~h}$ post feeding (Fig. 4e).

To further confirm that necroptosis is unlikely to be responsible to the initial cell death in the CDE model, knockout (KO) mice lacking in Mlkl, a terminal executor of necroptosis, were fed with a CDE diet at $0 \mathrm{~h}$, and then sacrificed at $18 \mathrm{~h}$ post feeding for further analyses. The measurement of serum injury markers and PI staining revealed that hepatic necrosis occurred in Mlkl KO as well as WT mice similarly, strongly suggesting that necroptosis is not likely to be the initial cell death in this model 


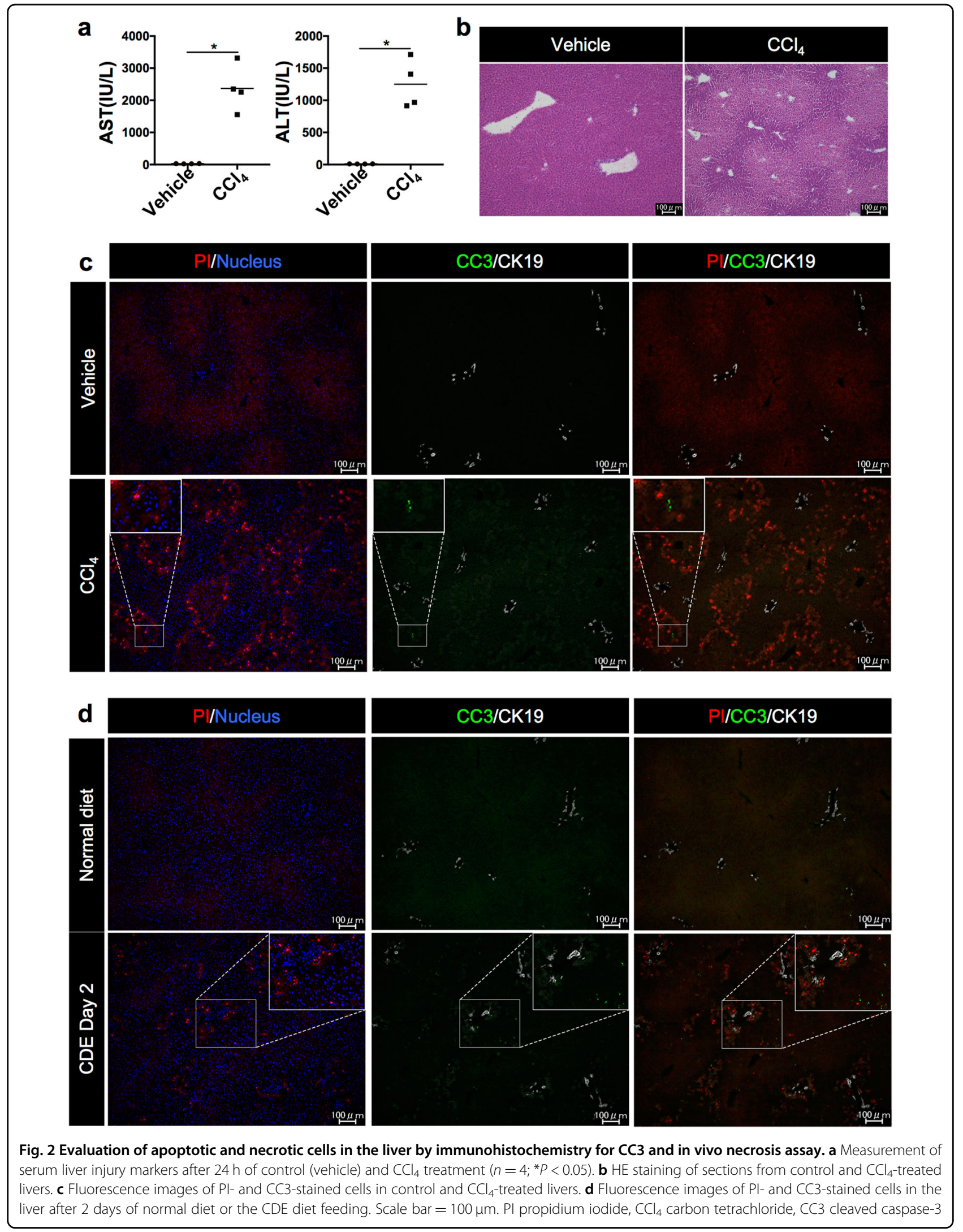


a

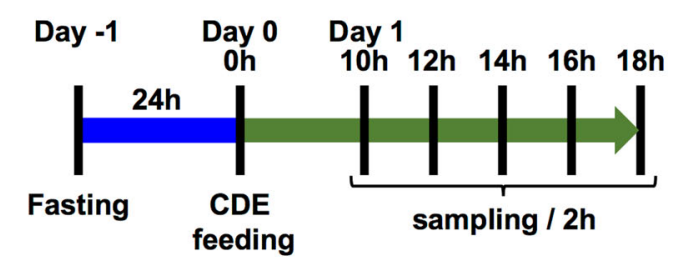

b

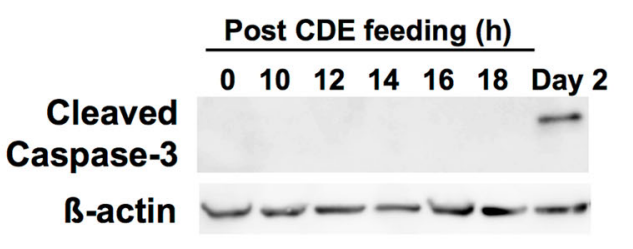

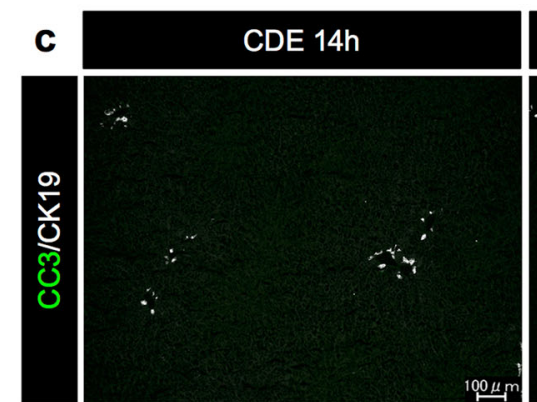
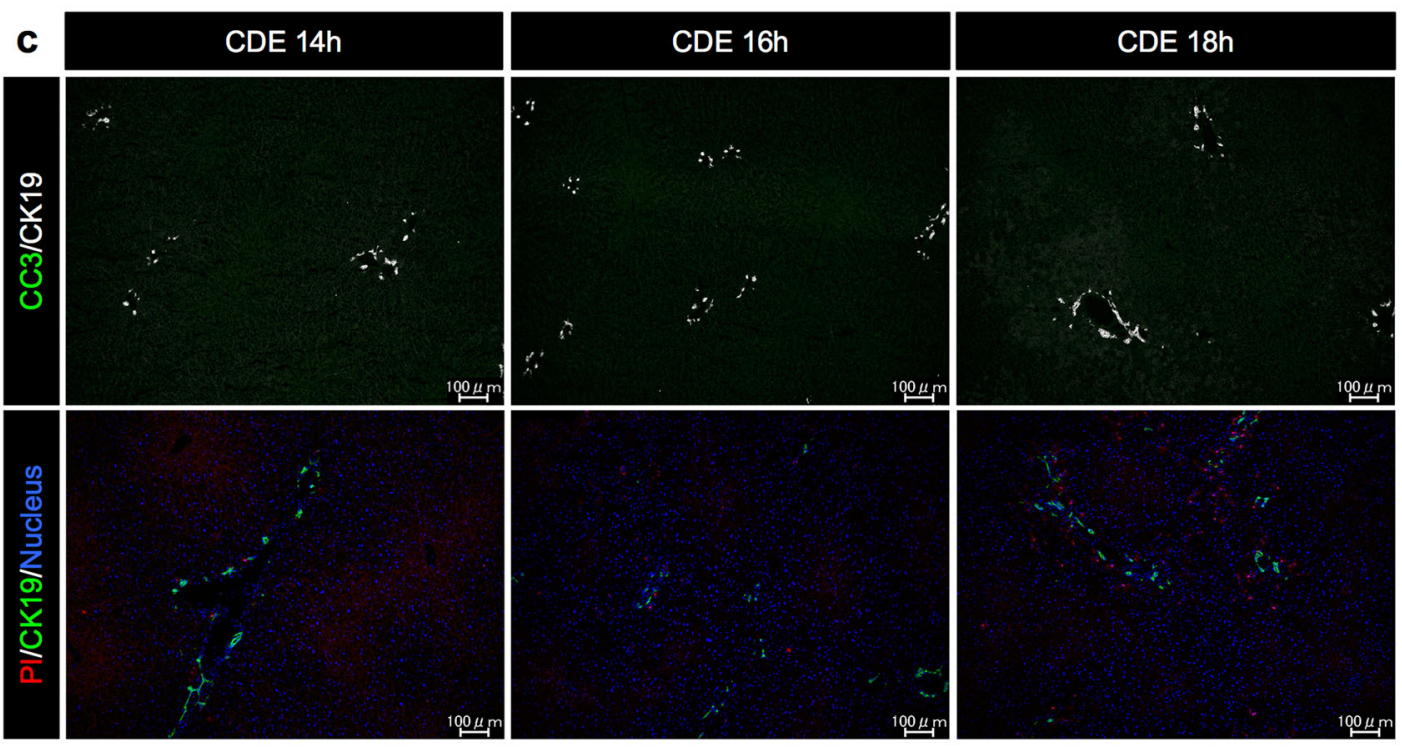

d
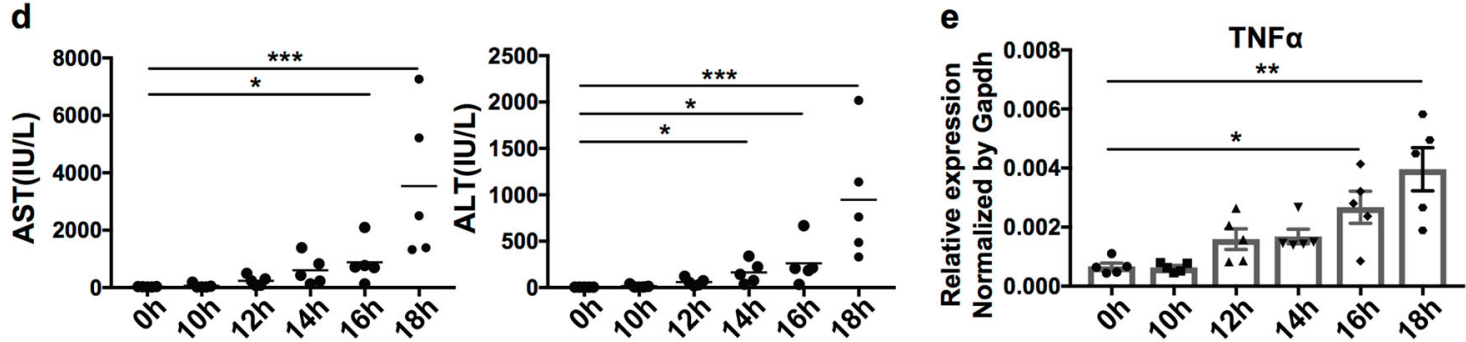

Fig. 3 Detection of apoptosis and necrosis at an early stage of steatohepatitis in the CDE diet model. a Experimental design for identifying apoptosis and necrosis at the onset of steatohepatitis. $\mathbf{b}$ Western blot analysis of CC3 protein in the liver extracts after the CDE diet feeding. B-Actin was used as an internal control. c Detection of apoptotic and necrotic cells in the liver after 14 to $18 \mathrm{~h}$ post CDE diet feeding by immunohistochemistry for CC3 and the in vivo necrosis assay. In images of apoptosis detection, CK19 is shown as white pseudocolor. d Measurement of serum liver injury markers after CDE diet feeding. $\left(n=5 ;{ }^{*} P<0.05,{ }^{* *} P<0.001\right)$. e Gene expression analysis of TNFa after the CDE diet feeding by quantitative RT-PCR. All data are normalized to Gapdh and shown as the mean \pm SEM $\left(n=5 ;{ }^{*} P<0.05,{ }^{* *} P<0.01\right)$. Scale bar $=100 \mu \mathrm{m}$

(Fig. 5a, b). However, Mlkl KO mice showed a significant down-regulation of gene expression in some inflammatory cytokines (Fig. 5c), suggesting that necroptosis of nonhepatocyte (e.g. immune cells) may be involved in the exacerbation of inflammation at the later stage of steatohepatitis.

Ferroptosis is a promising candidate for the initiating cell death in steatohepatitis

The accumulation of iron in the liver is considered as an aggravating factor of $\mathrm{NASH}^{22,23}$. Therefore, we have focused on ferroptosis as a possible initiator of steatohepatitis. Similar to the administration of Nec-1s into the CDE-fed mice, the effect of rosiglitazone (ROSI), an inhibitor of ACSL4, and Trolox, an antioxidant vitamin E analog, was examined, respectively. Both agents have been reported to inhibit ferroptosis previously ${ }^{10,13}$. The administration of ROSI showed a tendency towards amelioration of hepatic cell death judging from serum injury markers at $18 \mathrm{~h}$ post CDE feeding (Supplementary Figure S4). On the other hand, the administration of Trolox dramatically decreased the level of serum liver 


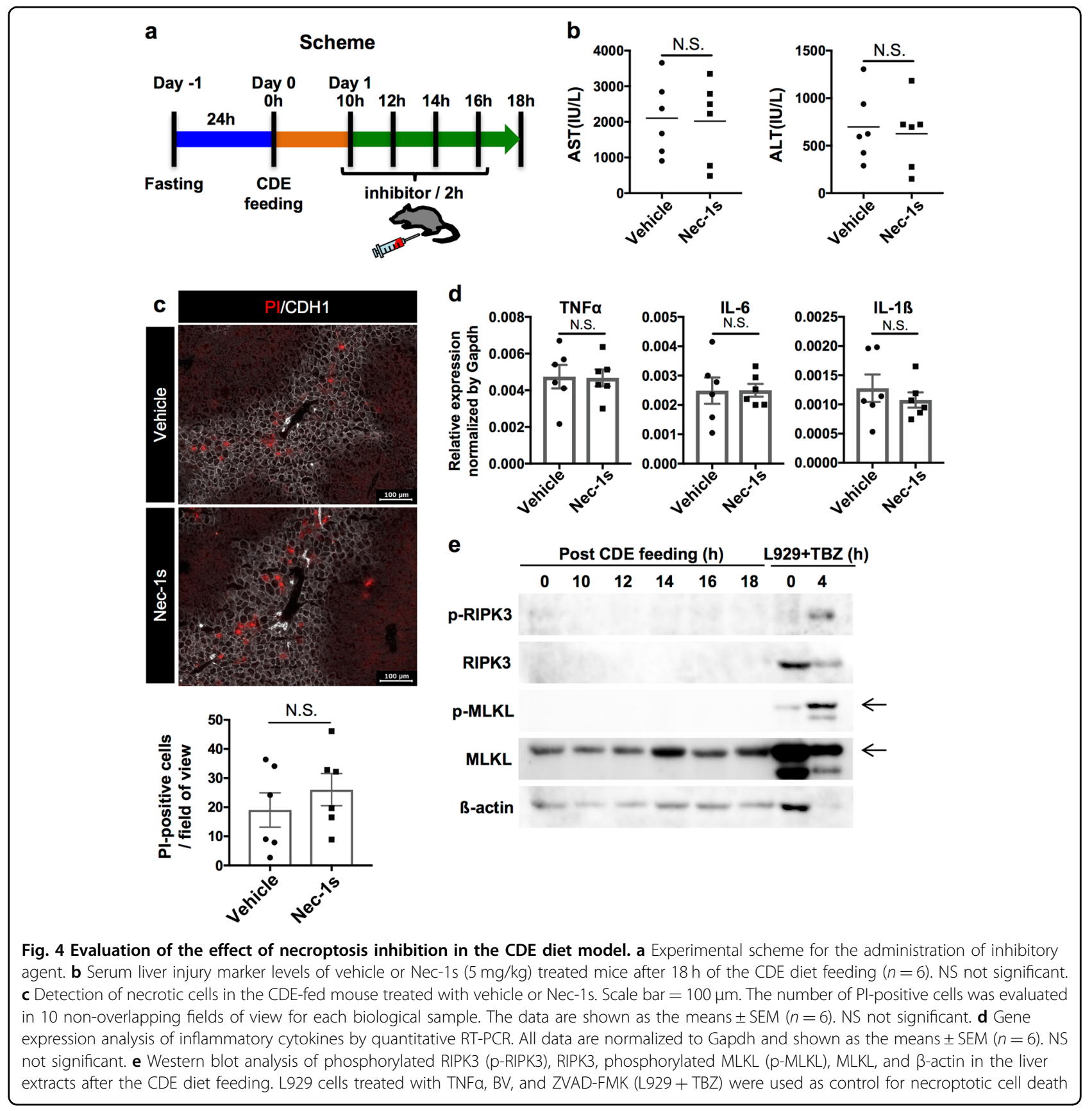

damage markers and the number of PI-positive necrotic cells even at $18 \mathrm{~h}$ post feeding (Fig. 6a, b). Also, gene expression of inflammatory cytokines and infiltration of inflammatory cells was significantly suppressed (Fig. 6c, d). Next, we compared the amount of phospholipid peroxides in the liver between control and Trolox-treated mice, because oxygenated PE has been recently reported to be involved in the pathway for ferroptotic signaling ${ }^{12,13}$. As a result, the amount of oxygenated PE was increased in the liver of CDE diet-fed mice compared with that of normal chow-fed mice. As expected, together with the amelioration of liver damage, the elevated amount of oxygenated PE in the livers of CDE-fed mice was ameliorated by the administration of Trolox (Fig. 6e). These results strongly suggested that ferroptosis is implicated in the pathogenesis of NASH.

\section{Effect of iron chelating agents on the suppression of initial necrosis in steatohepatitis}

To confirm whether ferroptosis is the trigger for steatohepatitis, we examined the effect of iron chelating agents, which are another type of ferroptosis inhibitor. 

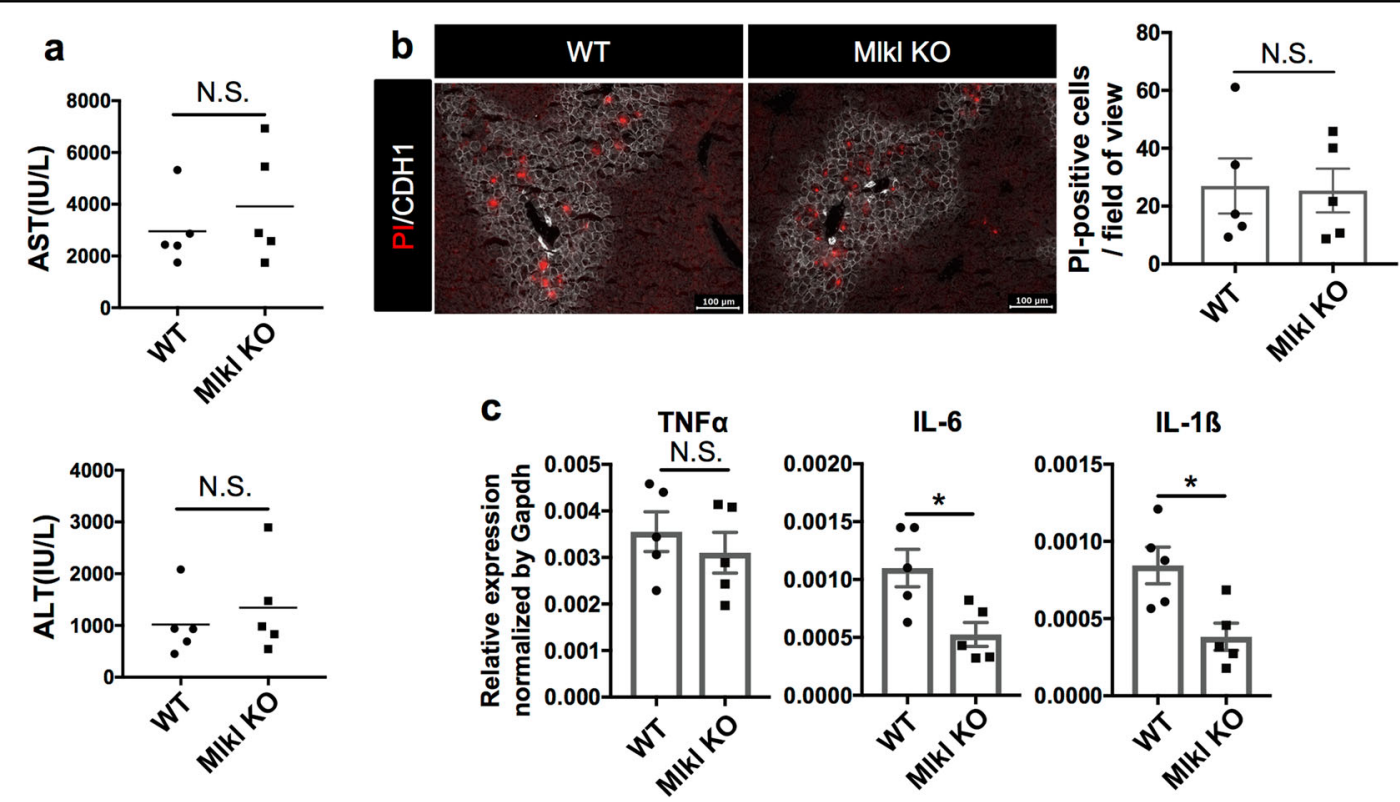

Fig. 5 Evaluation of necrotic cell and inflammation in the liver of CDE-fed WT and MIkl KO mice. a Serum liver injury marker levels of WT and MIkI KO mice after $18 \mathrm{~h}$ of the CDE diet feeding $(n=5)$. NS not significant. $\mathbf{b}$ Detection of necrotic cells in the liver of WT and Mlkl KO mice at $18 \mathrm{~h}$ post CDE feeding by the in vivo necrosis assay. The number of PI-positive cells was evaluated in 10 non-overlapping fields of view for each biological sample. The data are shown as the means \pm SEM $(n=5)$. NS not significant. c Gene expression analysis of inflammatory cytokines by quantitative RTPCR. All data are normalized to Gapdh and shown as the means \pm SEM $\left(n=5 ;{ }^{*} P<0.05\right)$

Iron is known to play a catalytic role for the fenton reaction, which promotes the production of lipid peroxides required for ferroptosis. Because Deferoxamine (DFO) is commonly used in the study of ferroptosis, we administered DFO to the CDE-fed mice. Contrary to our expectation, the administration of DFO did not ameliorate the increase of serum liver injury markers (Fig. $7 a)$, suggesting that the initial hepatic necrosis was not suppressed by DFO treatment. Interestingly, however, the administration of another iron chelator Deferiprone (DFP), which has higher membrane permeability than $\mathrm{DFO}^{24}$, markedly suppressed the elevation of serum liver injury markers and the emergence of PI-positive necrotic cells almost completely (Fig. 7b, c). As was the case with Trolox, infiltration of immune cells and inflammatory cytokine level were also suppressed by DFP administration (Fig. 7d, e), suggesting that intracellular iron plays a crucial role in necrotic cell death rather than extracellular one in the liver. Taken together, these data strongly suggested that ferroptosis is the initiating cell death that induces inflammation at the onset of steatohepatitis.

\section{Discussion}

Cell death is a commonly observed event in most human diseases. While apoptosis is a regulated cell death accompanied by intracellular signaling $\operatorname{cascades}^{25}$, necrosis had been considered as unregulated or accidental cell death. However, the discovery of a regulated form of necrotic cell death, namely "programmed necrosis" brought about a change in our view of necrosis, and much effort has been made to elucidate the molecular mechanisms underlying the execution of necrotic cell death ${ }^{10,26,27}$. Along with the advance in understanding of each programmed cell death, much attention has been paid to the pathophysiological roles of multiple types of cell death in a wide range of diseases including hepatic failure. Unlike the detection method of apoptosis such as TUNEL or CC3 staining, the difficulty of identifying necrosis in vivo has hampered the acquisition of regional information about apoptosis and necrosis in a wide area of injured tissue. In this study, we designed a new strategy by applying the PI staining for in vivo analysis and showed that initial cell death occurred predominantly in the periportal area at the onset of steatohepatitis. This method may be applicable to the other tissues, organs, or disease models.

In the pathogenesis of $\mathrm{NASH}$, there is accumulating evidence for the involvement of apoptosis in disease progression. The cytokeratin-18 fragment, which is a molecule cleaved by caspase and released from apoptotic hepatocytes, is used as one of the diagnostic biomarkers in NASH patients ${ }^{28,29}$. Although apoptosis inhibition is known to suppress fibrosis in murine NASH models, serum ALT levels are less affected, suggesting that the 

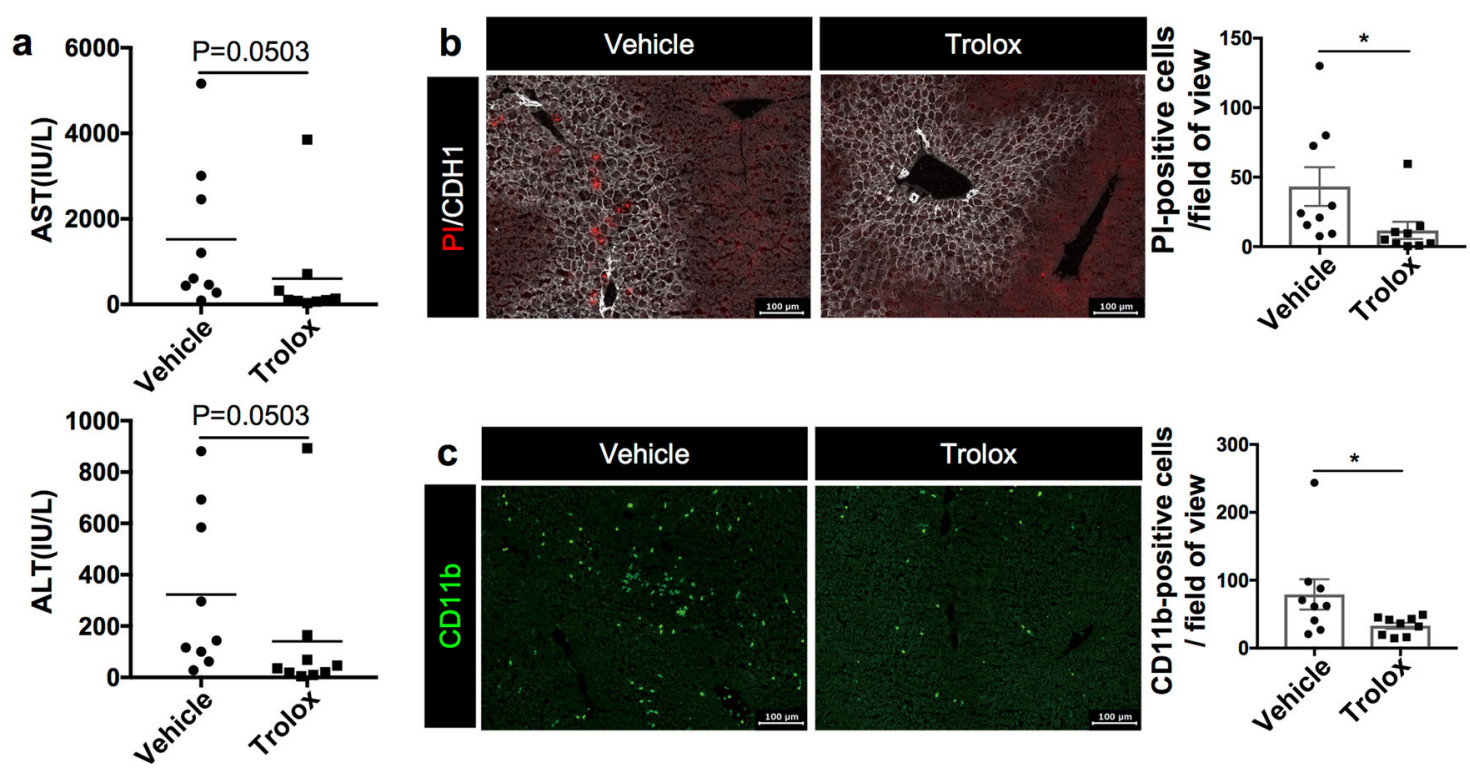

d
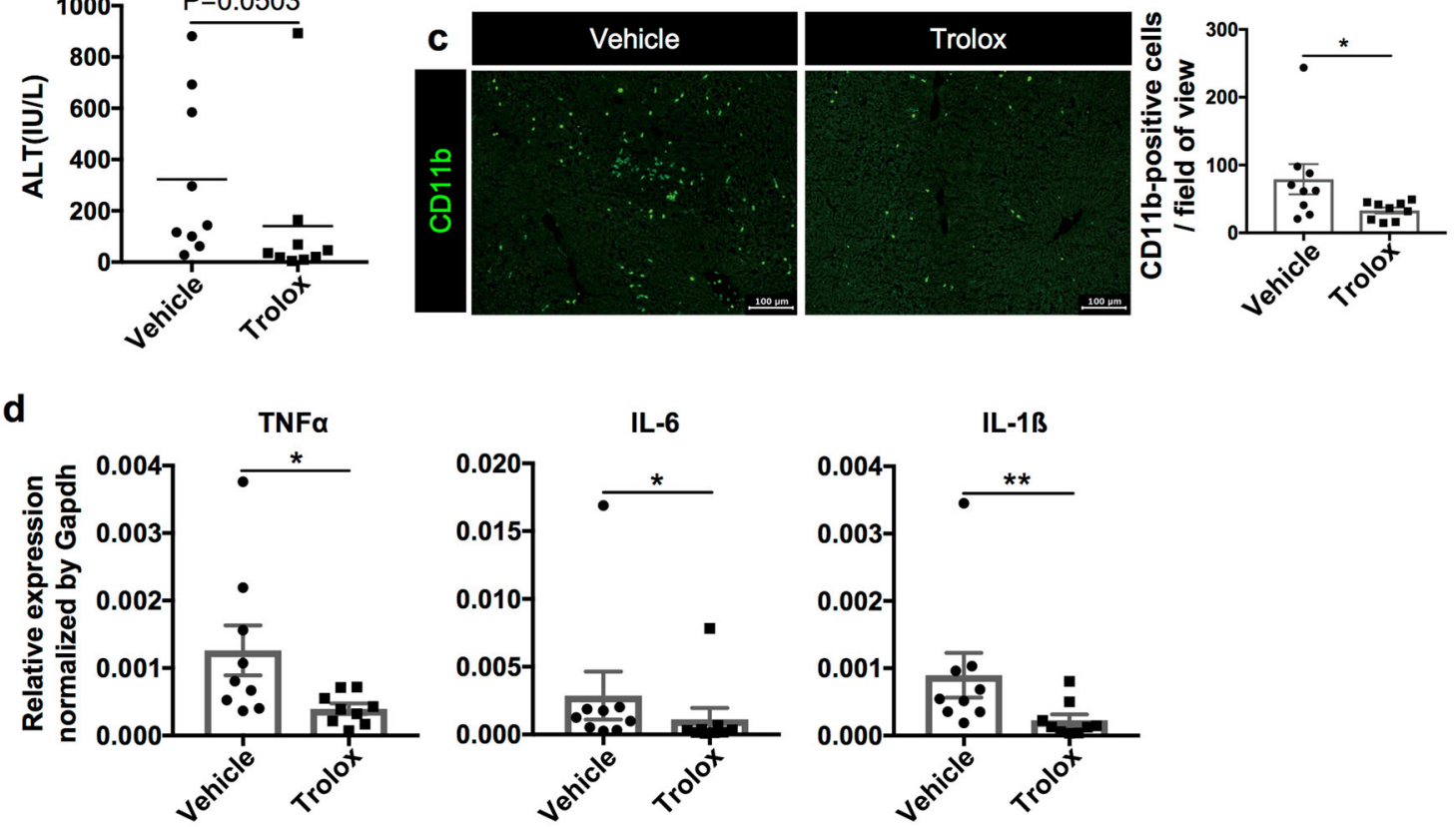

e

PE 16:0/18:2OOH

PE 18:0/18:200H
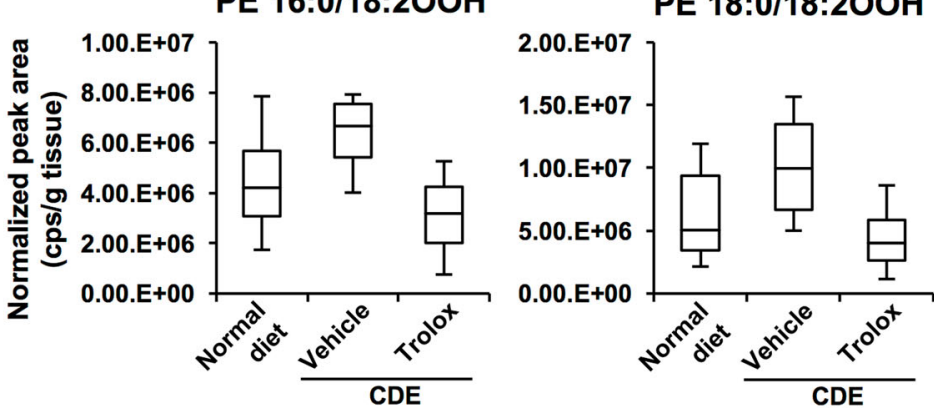

PE 18:1/18:2OOH

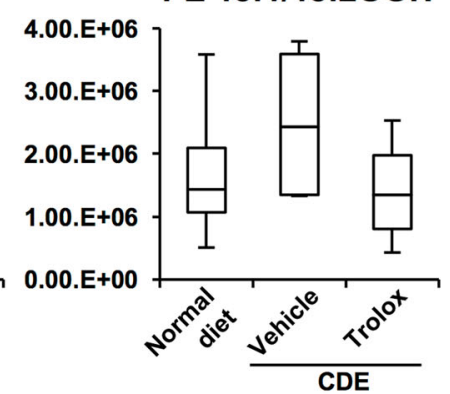

Fig. 6 Evaluation of the effect of ferroptosis inhibitor, Trolox, in the CDE diet model. a Serum liver injury marker levels in control (vehicle) or Trolox $(100 \mathrm{mg} / \mathrm{kg})$ treated mice $(n=9)$. b Detection of necrosis in the CDE-fed mice treated with vehicle or Trolox by the in vivo necrosis assay. The number of PI-positive cells was evaluated in 10 non-overlapping fields of view for each biological sample. The data are shown as the means \pm SEM $(n$ $=9$; $\left.{ }^{*} P<0.05\right)$. $\mathbf{c}$ Immunohistochemical analysis of CDE-fed mouse with vehicle or Trolox for CD11b. The number of CD11b-positive cells was evaluated in 10 non-overlapping fields of view for each mouse. The data are shown as the means \pm SEM $(n=9 ; * P<0.05)$. $\mathbf{d}$ Gene expression analysis of inflammatory cytokines by quantitative RT-PCR $(n=9)$. All data are normalized to Gapdh and shown as the means \pm SEM $\left(n=9 ;{ }^{*} P<0.05,{ }^{* *} P<\right.$ 0.01). e Measurement of oxidized PEs in the liver. The level of each oxidized PE in the liver sample was compared among normal diet-fed mice, CDE diet-fed mice, and Trolox-treated CDE diet-fed mice $(n=4)$. Scale bar $=100 \mu \mathrm{m}$

other types of cell death are involved in the pathogenesis of $\mathrm{NASH}^{30}$. In fact, necroptosis has been recently reported to contribute to the progression of NASH. RIPK3 and
MLKL, which are key regulators of necroptosis, were upregulated in both murine NASH models and NASH patients $^{20}$, and necroptosis inhibition in Ripk3 $\mathrm{KO}$ mice 

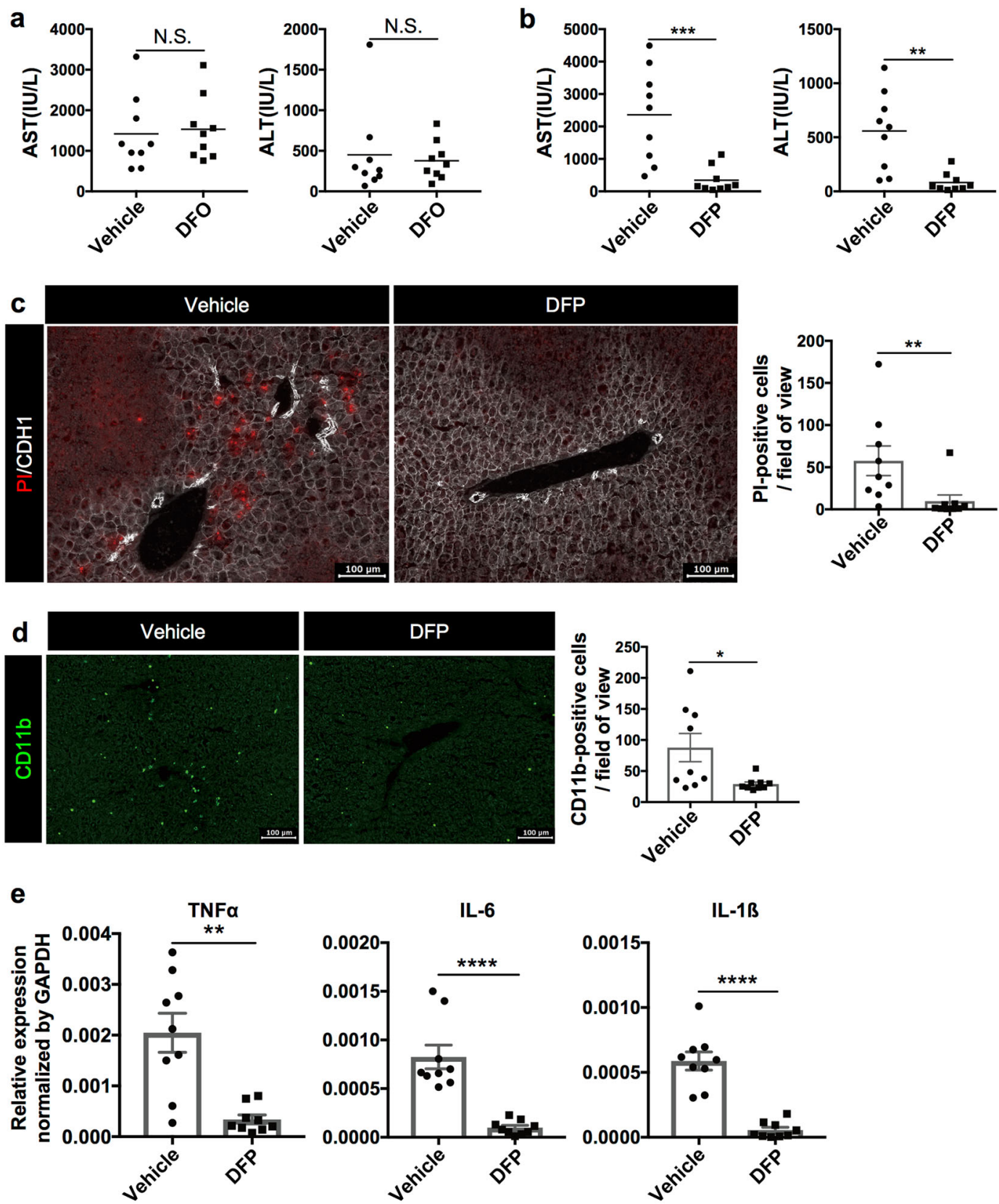

Fig. 7 Evaluation of the effect of iron chelators in the CDE diet model. a Serum liver injury marker levels in the CDE-fed mice treated with vehicle or DFO $(100 \mathrm{mg} / \mathrm{kg})(n=9)$. NS not significant. The serum data are shown as dot plots and mean. $\mathbf{b}$ Serum liver injury marker levels of the CDE-fed mice treated with vehicle or DFP $(100 \mathrm{mg} / \mathrm{kg})\left(n=9 ;{ }^{* *} P<0.01{ }^{* * *} P<0.001\right)$. c Detection of necrotic cells in the CDE-fed mice treated with vehicle or DFP. The number of PI-positive cells was evaluated in 10 non-overlapping fields of view for each biological sample. The data are shown as the means $\pm \operatorname{SEM}\left(n=9 ;{ }^{* *} P<0.01\right)$. d Immunohistochemical analysis of CDE-fed mouse treated with vehicle or DFP for CD11b. The number of CD11b-positive cells was evaluated in 10 non-overlapping fields of view for each biological sample. The data are shown as the mean $\pm \operatorname{SEM}\left(n=9\right.$; $\left.{ }^{*} P<0.05\right)$. e Gene expression analysis of inflammatory cytokines by quantitative RT-PCR. The gene expression data are normalized to Gapdh and shown as the means \pm $\operatorname{SEM}\left(n=9 ;{ }^{* *} P<0.01,{ }^{* * *} P<0.0001\right)$. Scale bar $=100 \mu \mathrm{m}$

shows decreased serum AST and ALT, inflammatory cytokines, and fibrosis ${ }^{5}$. More recently, pyroptosis, another type of programmed necrosis, has also been reported to play a role in the pathogenesis of NASH in
Gasdermin D KO mouse ${ }^{31}$. These studies strongly suggest that the pathological progression of NASH is governed by multiple types of cell death. However, their involvement in the initial hepatic cell death at the onset of 
steatohepatitis has remained unclear because it is difficult to specify when hepatocytes begin to die in fatty liver in long-term experimental settings.

On the other hand, ferroptosis is a recently identified programmed necrosis, which is an iron- and lipid hydroperoxide-dependent nonapoptotic cell death. For the pathogenesis of NASH, oxidative stress induced by the abnormal accumulation of lipid is considered as an important initiation factor ${ }^{32}$. There is further evidence to suggest the involvement of ferroptosis in NASH patients as follows: secondary products of lipid peroxidation such as malondialdehyde and 4-hydroxinonenal are utilized as oxidative stress markers in NASH patients ${ }^{33,34}$. Vitamin E, which is an antioxidant that suppresses peroxidizing lipids, reduces serum ALT in NASH patients ${ }^{35}$. Moreover, the iron accumulation resulting from metabolic aberration is also believed to be an aggravating factor because of the following reasons: liver siderosis is observed in some of NASH patients ${ }^{22}$. The iron removal by phlebotomy is known to ameliorate liver damage and serum $\mathrm{ALT}^{36}$. $\mathrm{NASH}$ is exacerbated in primary hemochromatosis patients $^{37}$. It is therefore plausible that ferroptosis is implicated in the pathogenesis of NASH. Nonetheless, there are few papers demonstrating the implication of ferroptosis in NASH. The reason why the possible role of ferroptosis has not been reported in murine model of NASH is as follows: the exact execution factors and signaling cascades of ferroptosis remain elusive, while those of apoptosis, necroptosis, and pyroptosis are well documented. Therefore, KO mice lacking in important components such as caspase family members, Ripk3, Mlkl, and Gsdmd, are useful for evaluating the contribution of the above three types of cell deaths to the pathogenesis of $\mathrm{NASH}^{5,30,31}$. In contrast, methods of ferroptosis inhibition in vivo are currently restricted to the use of several inhibitory agents such as antioxidants and iron chelators. Conventionally, in previous experiments using $\mathrm{KO}$ mice, the symptoms such as inflammation, fibrosis, and carcinogenesis have been examined after long-term feeding of MCD diet or high-fat $\operatorname{diet}^{38,39}$. Considering that the liver is the major organ of xenobiotic metabolism, continuous administration of chemical agents may not be suitable for follow-up examination in chronic liver injury models. Given that multiple types of cell death are involved in the progression of disease state in NASH, the effect of ferroptosis inhibition after the onset of steatohepatitis may become limited in conventional NASH models. Therefore, taking advantage of the CDE diet model, in which the hepatic cell death occurs within a short period of time in the context of steatosis, we focused on the initial cell death at the onset of steatohepatitis.

In this study, we demonstrated that necrosis precedes apoptosis at the early stage of steatohepatitis and that two ferroptosis inhibitors, Trolox and DFP, suppressed necrotic cell death, infiltration of inflammatory cells, and inflammatory cytokine expression almost completely. In addition, we showed that the amount of oxygenated PE, which is implicated in the ferroptosis pathway, was increased in the liver of the CDE-fed mice compared with normal diet-fed mice, and that the increase of oxygenated PE was lowered to a normal liver level by Trolox treatment. Consistently, it is reported that the hepatic PC/PE ratios are decreased in NASH patients ${ }^{40}$. These results suggested that ferroptosis is the initial necrosis to be the trigger for steatohepatitis.

By contrast, necroptosis inhibition by using Nec-1s or Mlkl KO mice could not block the initial necrotic cell death. However, Mlkl KO mice showed a tendency of decreasing inflammatory cytokine expression, implying that necroptosis in the non-parenchymal cells might be involved in the exacerbation of inflammation in NASH. Although it is unclear whether ferroptosis is followed by necroptosis and apoptosis in the progression stage of $\mathrm{NASH}$, there is evidence supporting the hypothesis that ferroptosis occurs in parallel with the other types of cell death in NASH patients as described above. Total inhibition of multiple cell deaths relevant to NASH pathogenesis could prove to be effective in the treatment of NASH. Therefore, elucidation of the correlation among multiple types of cell death in the pathological process of NASH is crucial.

In conclusion, our data suggested that ferroptosis plays a potential role as the trigger for steatohepatitis, leading to liver damage, infiltration of immune cells, and inflammatory reaction. Ferroptosis is a promising therapeutic target for the prevention of onset of steatohepatitis. Therefore, it is necessary to take ferroptosis into account for the therapeutic strategy of NASH. Our findings will provide a new insight into the type of cell death relevant to the pathogenesis of NASH.

\section{Materials and methods \\ Mice and models of steatohepatitis by the CDE diet and CDAA-based high-fat diet}

Male C57BL/6J mice were purchased from CLEA Japan, Inc. $M l k l-/-$ mice were provided by M. Pasparakis and described prebiously ${ }^{41}$. All animal experiments were conducted in accordance with institutional procedures and admitted by the Animal Care and Use committee of the Institute for Quantitative Biosciences, the University of Tokyo and Research Institute, the National Center for Global Health and Medicine. We used 5- to 6-week-old mice for feeding CDE diet (MP Biomedicals, USA) and CDAA-based high-fat diet (CDAHFD, A06071302, Research diet, USA), and harvested liver and blood samples for further analyses. 


\section{Measurement of serum ALT and AST}

The serum liver injury markers, ALT and AST were measured using Transaminase C-II Test Wako (Wako, Japan) according to the manufacturer's instruction.

\section{In vivo necrosis assay by $\mathrm{PI}$ injection}

PI (Sigma, USA) was dissolved in phosphate-buffered saline (PBS) at a final concentration of $25 \mu \mathrm{g} / \mathrm{ml}$. For the detection of necrotic cells in the liver, the PI solution was injected into mice intravenously via the tail vein. The liver samples were harvested and snap frozen in liquid nitrogen $10 \mathrm{~min}$ later. The frozen block was cut into slices with $8 \mu \mathrm{m}$ thickness by using Microtome Cryostat HM 525 (Thermo Fisher Scientific Industries, Osaka, Japan) and mounted on slide glasses. After imaging of PI-stained tissues, the samples were counterstained with Hoechst, anti-Cleaved caspase-3 (CC3, \#9446, CST, USA, 1:300), anti-Cadherin1 (CDH1, \#3195, CST, USA, 1:200) and anti-CK19 (DSHB, USA, 1:1000) antibodies. Merged images were captured using BZ-X710:BZ-X Viewer (KEYENCE, Japan). The number of PI-positive cells was counted using Hybrid Cell Count function in the Dual Signal Extraction mode of BZ-X Analyzer. An average value of 10 random images per mouse was treated as a representative value for the mouse.

\section{$\mathrm{CCl}_{4}$ liver injury model}

We injected $\mathrm{CCl}_{4}$ dissolved in olive oil ( $2 \mathrm{mg} / \mathrm{kg}$; Wako) into 8-week-old mice intraperitoneally and harvested liver samples after $24 \mathrm{~h}$. Prior to the in vivo PI staining, we collected blood samples for measurement of serum AST and ALT.

\section{Inhibition of cell death in the CDE-fed liver by using inhibitors}

We used 5- to 6-week-old mice for this experiment. After fasting for $24 \mathrm{~h}$, the CDE diet was fed at $0 \mathrm{~h}$. The inhibitor was injected intraperitoneally in four doses every $2 \mathrm{~h}$ from 10 to $16 \mathrm{~h}$ and then mice were sacrificed for evaluation at $18 \mathrm{~h}$. After the blood samples were collected, the in vivo necrosis assay was performed as described before. The final dose of used inhibitor used is as follows: Necrostatin- $1 \mathrm{~s} \mathrm{(} 5 \mathrm{mg} / \mathrm{kg}$; Focus Biomolecules, USA), Rosiglitazone (5 mg/kg; Sigma), Trolox (100 mg/ $\mathrm{kg}$; Sigma), Deferiprone (100 mg/kg; Sigma), and Deferoxamine $(100 \mathrm{mg} / \mathrm{kg}$; Sigma). For injection, Trolox was initially dissolved in a small amount of DMSO (Sigma), and then diluted in olive oil. Nec-1s and Rosiglitazone were first dissolved in DMSO, and then diluted in PBS. All other inhibitors were diluted in PBS. Similarly, wildtype (WT) and $M l k l \mathrm{KO}$ mice were analyzed as described above.

\section{$\mathrm{HE}$, Picro-Sirius Red, and Oil Red $\mathrm{O}$ staining}

Frozen sections of liver sample with $8 \mu \mathrm{m}$ thickness were fixed with $4 \%$ paraformaldehyde at room temperature for HE staining. For evaluation of liver fibrosis, PicroSirius Red staining was performed as described before ${ }^{42}$. For Oil Red O staining, the sections were stained with Mayer's hematoxylin solution (Wako) after rinsing with PBS. Then, the sections were washed with running water, incubated in 60\% isopropanol (Wako), stained with Oil Red O staining solution (SIGMA), and reincubated in $60 \%$ isopropanol. Finally, the sections were washed with running water and mounted with fluoromount (Cosmo Bio, Japan).

\section{Immunohistochemistry}

For immunohistochemistry, fixed samples with $4 \%$ paraformaldehyde were blocked with PBS containing 1\% bovine serum albumin and $0.3 \%$ Triton X-100, and incubated with anti-CC3, anti-CK19, and anti-CD11b (BD Pharmingen, USA; 1:200) antibodies at $4{ }^{\circ} \mathrm{C}$ overnight. The second antibodies conjugated with Alexa Fluor 488 or Alexa Fluor 647 (Thermo Fisher Scientific, USA, 1:800) were used for immunofluorescence. All images of tissue sections were captured using BZ-X710:BZ-X Viewer (Keyence, Japan).

\section{Total RNA extraction and quantitative RT-PCR}

Total RNA was extracted from liver tissues with TRIzol reagent (Invitrogen, Carlsbad, CA, USA). The cDNA was synthesized from RNA with PrimeScript ${ }^{\mathrm{TM}}$ RT Master Mix (Takara, Japan) for quantitative RT-PCR. The expression level normalized to Gapdh was analyzed by using the LightCycler 480 (Roche, Basel, Switzerland). The primer sequences are listed in Table 1.

\section{Western blot analysis}

Proteins were extracted with RIPA lysis buffer from liver tissues and each concentration was measured by Bradford protein assay. Protein samples were electrophoresed on polyacrylamide gels and transferred onto PVDF membranes. Membranes were blocked with 5\% skim milk in PBS and incubated with anti-MLKL Rat Ab (\#MABC604; EMD Millipore, USA, 1:1000), antiPhospho-MLKL (Ser345) Rabbit Ab (Mouse specific) (\#62233; CST, USA, 1:1000), anti-Phospho-RIP3 (Thr231/Ser232) rabbit antibody (Mouse specific) (\#57220; CST, USA, 1:1000), anti-CC3 (\#9446; CST, USA, 1:500), and anti- $\beta$-actin (\#4967; CST, USA, 1:1000; 622101 Biolegend, Japan, 1:1000)) at $4{ }^{\circ} \mathrm{C}$ overnight. After washing with TBST, membranes were incubated with secondary antibody at room temperature for $60 \mathrm{~min}$ and washed with TBST. The immunoblot was then imaged 
Table 1 Primer sequences for quantitative RT-PCR

\begin{tabular}{lll}
\hline Genes & Forward primer sequence $\left(\mathbf{5}^{\prime} \boldsymbol{\rightarrow} \mathbf{3}^{\prime}\right)$ & Reverse primer sequence $\left(\mathbf{5}^{\prime} \boldsymbol{\rightarrow} \mathbf{3}^{\prime}\right)$ \\
\hline TNFa & TCTTCTCATTCCTGCTTGTGG & GGTCTGGGCCATAGAACTGA \\
IL-6 & GCTACCAAACTGGATATAATCAGGA & CCAGGTAGCTATGGTACTCCAGAA \\
IL-1ß & AGTGACGGACCCCAAAAG & AGCTGGATGCTCTCATCAGG \\
TGFß & TGGAGCAACATGTGGAACTC & CAGCAGCCGGTTACCAAG \\
Collagen 1a1 & GATGTTCAGCTTGTGGACCT & GCAGCTGACTTCAGGGATGT \\
Collagen 3a1 & TCCCCTGGATCTGTGAATC & TGAGTCGAATTGGGAGAAT \\
\hline
\end{tabular}

according to the manufacturer's instructions from Clarity $^{\mathrm{TM}}$ Western ECL Substrate (Bio Rad, USA), and semiquantitatively measured by Multi Gauge (Fuji Film, Japan). For the preparation of necroptotic sample, L929 cells were cultured in the presence of $20 \mu \mathrm{M}$ ZVAD-FMK for $30 \mathrm{~min}$, followed by the addition of $20 \mathrm{ng} / \mathrm{ml}$ of mouse TNF $\alpha$ and $1 \mu \mathrm{M}$ BV. After $4 \mathrm{~h}$ of culture, the cell lysate was recovered for western blotting.

\section{Sample preparation for LC-MS}

For lipid extraction, the liver of mice $(0.1 \mathrm{~g})$ were homogenized with $1 \mathrm{ml}$ methanol including an internal standard (17:0-lysoPC) using a glass homogenizer and extracted after stand for $1 \mathrm{~h}$ at on ice. The samples were centrifuged (7000 r.p.m., $4{ }^{\circ} \mathrm{C}, 5 \mathrm{~min}$ ) to remove cellular and protein materials, then the supernatant was diluted with 10 volumes of water and then adjusted to $\mathrm{pH} 3.0$ with $0.1 \mathrm{~N} \mathrm{HCl}$. The samples were applied to preconditioned (20 ml of methanol and $20 \mathrm{ml}$ of water) C18 SepPak cartridges (500 mg; Waters, Millford, MA, USA), and washed with $20 \mathrm{ml}$ of water to exclude nonvolatile ions followed by $10 \mathrm{ml}$ of hexane to exclude cholesterols and neutral lipids. The samples were eluted with $10 \mathrm{ml}$ of methanol to obtain oxidized phospholipids. The lipid extracts were dried under a gentle stream of nitrogen, dissolved in $1 \mathrm{ml}$ methanol, and stored at $-80{ }^{\circ} \mathrm{C}$ until use.

\section{LC-ESI-MS/MS system}

The LC-ESI-MS/MS analysis was carried out using a QTRAP 4500 quadropole linear ion trap hybrid mass spectrometer (AB Sciex, Concord, ON, Canada) with a Nexera XR high-performance liquid chromatography (Shimadzu Co., Kyoto, Japan). The sample was subjected to LC-ESI-MS/MS analysis using the XBridge BEH C18 column (Waters). Sample was injected by the autosampler, and the phospholipid fractions were separated by a step gradient with mobile phase A (acetonitrile/methanol $/$ water $=2: 2: 1 \mathrm{v} / \mathrm{v} / \mathrm{v}$ containing $0.1 \%$ formic acid and $0.028 \%$ ammonia): mobile phase B (isopropanol containing $0.1 \%$ formic acid and $0.028 \%$ ammonia) ratios of 100:0
(0-5 min), 50:50 (5-25 min), 50:50 (25-59 min), 100:0 (59-60 $\mathrm{min})$, and 100:0 (60-75 $\mathrm{min})$ at a flow rate of $70 \mu \mathrm{L} / \mathrm{min}$ and a column temperature of $30^{\circ} \mathrm{C}$. The multiple reaction monitoring was carried out to detect specific oxidized phospholipids. MS/MS analysis was carried out in negative ion mode with the following settings, ion spray voltage, $-4500 \mathrm{~V}$; curtain gas (N2), 30 arbitrary units; collision gas (N2), "medium"; declustering potential, -60 to $-170 \mathrm{~V}$, collision energy, -40 to $-44 \mathrm{eV}$; temperature, $500{ }^{\circ} \mathrm{C}$. For the detection of $\mathrm{PEOOH}$, deprotonated ions $\left([\mathrm{M}-\mathrm{H}]^{-}\right)$were selected as a precursor ion, and the peroxidized fatty acyl chain were selected as product ions $\left(\left[\mathrm{M}-\mathrm{H}-\mathrm{H}_{2} \mathrm{O}\right]^{-}\right)$.

\section{Statistical analyses}

Statistical analyses were performed using GraphPad Prism software. Statistical significance between two groups was evaluated using a two-tailed Mann-Whitney $U$-test. For comparison of more than three groups, Kruskal-Wallis test was applied. $P<0.05$ was considered statistically significant. The exact number of biological samples was described in each figure legend. There was no exclusion of outliers in all experiments. Group allocation was performed without any bias.

\footnotetext{
Acknowledgements

We would like to thank Dr. Manolis Pasparakis (University of Cologne, Germany) for providing MIkl-/- mice, and Dr. Mikiko Sodeoka and Kosuke Dodo (Riken, Saitama, Japan) for supplying chemical inhibitors. We also thank the members of the Miyajima and Okochi laboratory for discussion and advices. This work was supported by Japan Society for the Promotion of Science (JSPS) KAKENHI Grants JP26110007 (to M.T.), JP26110003 (to H.N.), JP26253023 (to A.M.), JP15H01386 and JP17H05513 (to H.I.); Japan Agency for Medical Research and Development (AMED) under Grant Number JP18gm1210002 (to M.T. and H.N.) and JP18gm0910013 (to H.I.).
}

\section{Author details}

${ }^{1}$ Department of Regenerative Medicine, Research Institute, National Center for Global Health and Medicine, Tokyo, Japan. '2Laboratory of Stem Cell Regulation, Institute for Quantitative Biosciences, The University of Tokyo, Tokyo, Japan. ${ }^{3}$ Department of Biochemistry, Toho University School of Medicine, Tokyo, Japan. ${ }^{4}$ School of Pharmaceutical Sciences, Kitasato University, Tokyo, Japan. ${ }^{5}$ Laboratory of Stem Cell Therapy, Institute for Quantitative Biosciences, The University of Tokyo, Tokyo, Japan. ${ }^{6}$ Present address: Centre for Heart Research, The Westmead Institute for Medical Research, Westmead, NSW, Australia 


\section{Conflict of interest}

The authors declare that they have no conflict of interest.

\section{Publisher's note}

Springer Nature remains neutral with regard to jurisdictional claims in published maps and institutional affiliations.

Supplementary Information accompanies this paper at (https://doi.org/ 10.1038/s41419-019-1678-y).

Received: 14 January 2019 Revised: 4 May 2019 Accepted: 14 May 2019 Published online: 18 June 2019

\section{References}

1. Matteoni, C. et al. Nonalcoholic fatty liver disease: a spectrum of clinical and pathological severity. Gastroenterology 116, 1413-1419 (1999).

2. Yatsuji, S. et al. Clinical features and outcomes of cirrhosis due to non-alcoholic steatohepatitis compared with cirrhosis caused by chronic hepatitis C. J. Gastroenterol. Hepatol. 24, 248-254 (2009).

3. Takaki, A., Kawai, D. \& Yamamoto, K. Molecular mechanisms and new treatment strategies for non-alcoholic steatohepatitis (NASH). Int. J. Mol. Sci. $\mathbf{1 5}$ 7352-7379 (2014).

4. Barreyro, F. J. et al. The pan-caspase inhibitor Emricasan (IDN-6556) decreases liver injury and fibrosis in a murine model of non-alcoholic steatohepatitis. Liver Int. 35, 953-966 (2015).

5. Gautheron, J. et al. A positive feedback loop between RIP3 and JNK controls non-alcoholic steatohepatitis. EMBO Mol. Med. 6, 1062-1074 (2014).

6. Köhn-Gaone, J. et al. Divergent inflammatory, fibrogenic, and liver progenitor cell dynamics in two common mouse models of chronic liver injury. Am. J. Pathol. 186, 1762-1774 (2016).

7. Jha, P. et al. Role of adipose tissue in methionine-choline-deficient model of non-alcoholic steatohepatitis (NASH). Biochim. Biophys. Acta 1842, 959-970 (2014).

8. Kirsch, R. et al. Rodent nutritional model of non-alcoholic steatohepatitis: species, strain and sex difference studies. J. Gastroenterol. Hepatol. 18, 1272-1282 (2003)

9. Yang, Y. M. et al. Interventional potential of recombinant feline hepatocyte growth factor in a mouse model of non-alcoholic steatohepatitis. Front. Endocrinol. (Lausanne) 9, 1-10 (2018).

10. Dixon, S. J. et al. Ferroptosis: an iron-dependent form of nonapoptotic cell death. Cell 149, 1060-1072 (2012).

11. Yang, W. S. et al. Regulation of ferroptotic cancer cell death by GPX4. Cell 156, 317-331 (2014).

12. Kagan, V. E. et al. Oxidized arachidonic and adrenic PEs navigate cells to ferroptosis. Nat. Chem. Biol. 13, 81-90 (2017).

13. Doll, S. et al. ACSL4 dictates ferroptosis sensitivity by shaping cellular lipid composition. Nat. Chem. Biol. 13, 91-98 (2017).

14. Hambright, W. S., Fonseca, R. S., Chen, L., Na, R. \& Ran, Q. Ablation of ferroptosis regulator glutathione peroxidase 4 in forebrain neurons promotes cognitive impairment and neurodegeneration. Redox Biol. 12, 8-17 (2017).

15. Friedmann Angeli, J. P. et al. Inactivation of the ferroptosis regulator Gpx4 triggers acute renal failure in mice. Nat. Cell Biol. 16, 1180-1191 (2014).

16. Miyajima, A., Tanaka, M. \& Itoh, T. Stem/progenitor cells in liver development, homeostasis, regeneration, and reprogramming. Cell Stem Cell 14, 561-574 (2014).

17. Matsumoto, M. et al. An improved mouse model that rapidly develops fibrosis in non-alcoholic steatohepatitis. Int. J. Exp. Pathol. 94, 93-103 (2013).

18. Stachura, J. et al. Prostaglandin protection of carbon tetrachloride-induced liver cell necrosis in the rat. Gastroenterology 81, 211-217 (1981).
19. $\mathrm{Pu}, \mathrm{W}$. et al. Mfsd2a+hepatocytes repopulate the liver during injury and regeneration. Nat. Commun. 7, 13369 (2016).

20. Afonso, M. B. et al. Necroptosis is a key pathogenic event in human and experimental murine models of non-alcoholic steatohepatitis. Clin. Sci. 129, 721-739 (2015).

21. Zhao, J. et al. Mixed lineage kinase domain-like is a key receptor interacting protein 3 downstream component of TNF-induced necrosis. Proc. Natl. Acad. Sci. USA 109, 5322-5327 (2012).

22. Nelson, J. E. et al. Relationship between the pattern of hepatic iron deposition and histological severity in nonalcoholic fatty liver disease. Hepatology $\mathbf{5 3}$, 448-457 (2011).

23. O'Brien, J. \& Powell, L. W. Non-alcoholic fatty liver disease: is iron relevant? Hepatol. Int. 6, 332-341 (2012).

24. Kartikasari, A. E. R. et al. Intracellular labile iron modulates adhesion of human monocytes to human endothelial cells. Arterioscler. Thromb. Vasc. Biol. 24 2257-2262 (2004).

25. Elmore, S. Apoptosis: a review of programmed cell death. Toxicol. Pathol. 35, 495-516 (2007).

26. Vercammen, D. et al. Inhibition of caspases increases the sensitivity of L929 cells to necrosis mediated by tumor necrosis factor. J. Exp. Med. 187, 1477-1485 (1998).

27. Brennan, M. A. \& Cookson, B. T. Salmonella induces macrophage death by caspase-1-dependent necrosis. Mol. Microbiol. 38, 31-40 (2000).

28. Eguchi, A., Wree, A. \& Feldstein, A. E. Biomarkers of liver cell death. J. Hepatol. 60, 1063-1074 (2014).

29. Vuppalanchi, R. et al. Relationship between changes in serum levels of keratin 18 and changes in liver histology in children and adults with nonalcoholic fatty liver disease. Clin. Gastroenterol. Hepatol. 12, 2121-2130 (2014).

30. Thapaliya, S. et al. Caspase 3 inactivation protects against hepatic cell death and ameliorates fibrogenesis in a diet-induced NASH model. Dig. Dis. Sci. 59, 1197-1206 (2014)

31. $\mathrm{Xu}$, B. et al. Gasdermin D plays a key role as a pyroptosis executor of nonalcoholic steatohepatitis in humans and mice. J. Hepatol. 68, 773-782 (2018).

32. MacHado, M. V. \& Diehl, A. M. Pathogenesis of nonalcoholic steatohepatitis. Gastroenterology 150, 1769-1777 (2016).

33. Casoinic, F., Sampelean, D., Buzoianu, A. D., Hancu, N. \& Baston, D. Serum levels of oxidative stress markers in patients with type 2 diabetes mellitus and nonalcoholic steatohepatitis. Rom. J. Intern. Med 54, 228-236 (2016).

34. Loguercio, C. et al. Non-alcoholic fatty liver disease in an area of southern Italy: main clinical, histological, and pathophysiological aspects. J. Hepatol. 35, 568-574 (2001).

35. Lavine, J. E., Ph, D. \& Tonascia, J. Pioglitazone, vitamin E, or placebo for nonalcoholic steatohepatitis. N. Engl. J. Med. 362, 1675-1685 (2010).

36. Valenti, L. et al. Venesection for non-alcoholic fatty liver disease unresponsive to lifestyle counselling-a propensity score-adjusted observational study. QJM 104, 141-149 (2011).

37. Bonkovsky, H. L. et al. Non-alcoholic steatohepatitis and iron: increased prevalence of mutations of the HFE gene in non-alcoholic steatohepatitis. J. Hepatol. 31, 421-429 (1999).

38. Horie, Y. et al. Hepatocyte-specific Pten deficiency results in steatohepatitis and hepatocellular carcinomas. J. Clin. Invest. 113, 1-20 (2012).

39. Itoh, M. et al. Melanocortin 4 receptor-deficient mice as a novel mouse model of nonalcoholic steatohepatitis. Am. J. Pathol. 179, 2454-2463 (2011).

40. Li, Z. et al. The ratio of phosphatidylcholine to phosphatidylethanolamine influences membrane integrity and steatohepatitis. Cell Metab. 3, 321-331 (2006).

41. Dannappel, M. et al. RIPK1 maintains epithelial homeostasis by inhibiting apoptosis and necroptosis. Nature 513, 90-94 (2014).

42. Yagai, T., Miyajima, A. \& Tanaka, M. Semaphorin 3E secreted by damaged hepatocytes regulates the sinusoidal regeneration and liver fibrosis during liver regeneration. Am. J. Pathol. 184, 2250-2259 (2014). 